\title{
Differential acuity of the two eyes
}

\author{
ANTONIO AUGUSTO VELASCO E CRUZ and HARLEY E. A. BICAS \\ University of São Paulo, Ribeirão Prêto, Brazil
}

\author{
(José Aparecido da Silva, Sponsor)
}

\begin{abstract}
In order to characterize the phenomenon of differential acuity of the two eyes, the visual threshold (OD and OS) of 180 individuals was measured using a scale whose angular variations were of the same magnitude and equal to $0.05^{\prime}$. The group was within the age range of 10 to 40 years. All subjects had normal ophthalmological examinations, were approximately isometropic, and had refractive errors not exceeding 3 spherical diopters or 1.5 cylindrical diopters. Measurements were made under optimal conditions in the same place and by a single examiner. This permitted obtaining a distribution of 180 acuities for OD and OS. The differences in resolving power detected between OD and OS for each individual were very small. In fact, even resolving power (null differences) was $45 \%$, and visual differences did not exceed $0.10^{\prime}$ in $95.5 \%$ of eye pairs. Among individuals whose eyes showed different resolving powers (55\% of the sample), there was no correlation between visual acuity and motor sighting dominance.
\end{abstract}

The expression "differential acuity of the two eyes" is used when the resolving powers of a person's two eyes are not the same. This phenomenon, also called "acuity dominance"' (Coren \& Kaplan, 1971), has been mainly investigated in the area of perception and psychophysics. Studies on differential acuity of the two eyes are always aimed at determining whether the difference in resolving power of the eye is associated with sighting dominance, that is, whether the dominant eye also has the best acuity. This question is still controversial, with some investigators denying such an association (Gahagan, 1933; Hebben, Benjamins, \& Milberg, 1981; Walls, 1951) and others proposing it (Crovitz, 1960; Porac, Whitford, \& Coren, 1976). With respect to this problem, a survey of the literature shows two important characteristics that we believe may be correlated. The first has to do with the discrepant results. Indeed, no emphatic statement has been provided thus far as to whether the difference in resolving power between the two eyes is associated with some other physiological variable. The second is the purely qualitative characteristic of these studies, with no report on the magnitude of the phenomenon of differential acuity of the two eyes. This is definitely an important point that reflects some methodological problems in the characterization of the differences in resolving power of the eyes. In fact, fundamental variables such as optic correction and type of acuity scale employed have not been properly considered.

Optic correction is of fundamental importance, since ametropias have a very strong effect on the measurement of visual acuity. Moreover, when a difference in resolving power is detected between left and right eyes, the

This research was presented at the 17th Annual Meeting of the Psychological Society of Ribeirão Prêto, October 1987. Requests for reprints should be sent to Antonio A. Velasco e Cruz, Department of Ophthalmology, University of São Paulo, 14049 Ribeirão Prêto, São Paulo, Brazil. characteristics of the scale used to make the measurement should be taken into account. For example, when using a visual acuity chart whose lowest angular value is $1^{\prime}$, the investigator will not be able to detect differences in visual threshold even when they are of the order of $0.5^{\prime}$ $(O D=1.0$ and $O S=2.0)$; therefore all eyes having $20 / 20$ acuity will be classified as cases of isoacuity (Gronwall \& Sampson, 1971). Furthermore, in commercial visual acuity charts the number and level of optotype contour interactions vary, with consequent greater imprecision of measurements (Romanio, 1971).

In view of the above considerations, the objective of the present study was to reexamine this subject by (1) characterizing the phenomenon of differential acuity of the two eyes from a quantitative viewpoint, that is, by determining the magnitude of the differences in resolving power detected between the eyes of normal in-

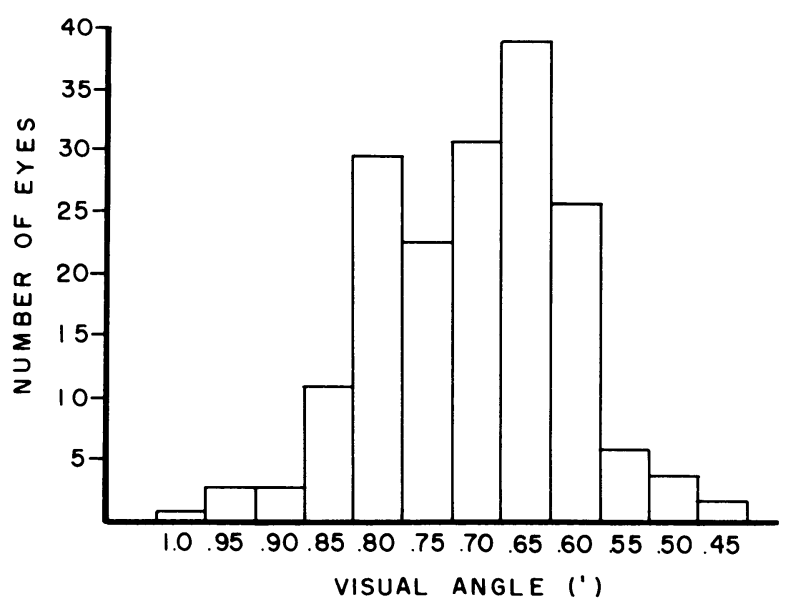

Figure 1. Distribution of OD visual angles among 180 normal subjects aged 10 to 40 years, who were optically corrected and approximately isometropic. 


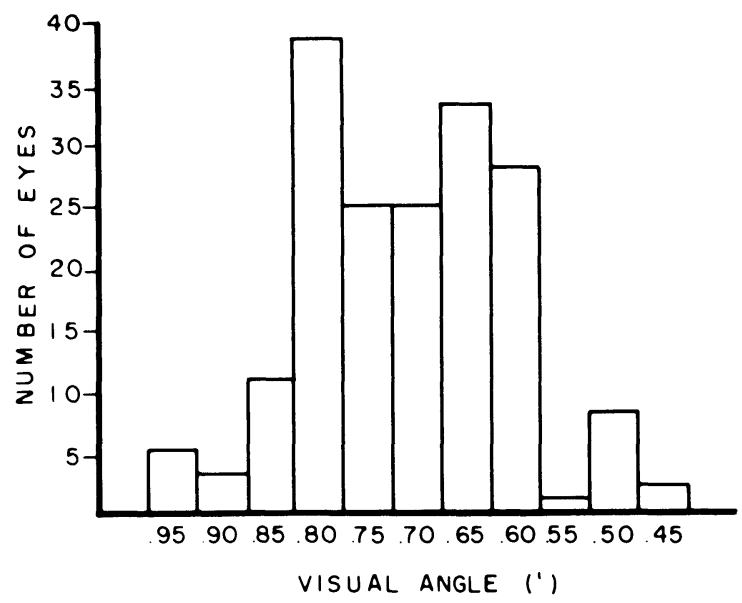

Figure 2. Distribution of $O S$ visual angles among 180 normal subjects aged 10 to 40 years, who were optically corrected and approximately isometropic.

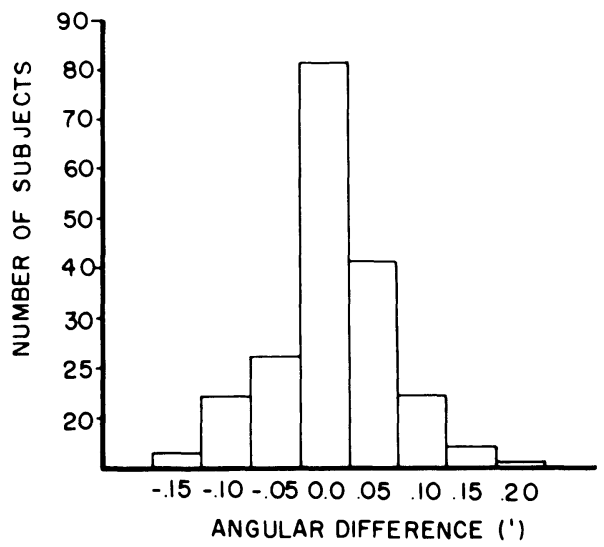

Figure 3. Distribution of the differences between $\mathrm{OS}$ and $\mathrm{OD}$ visual angles among 180 normal subjects aged 10 to 40 years, who were optically corrected and approximately isometropic.

dividuals, and (2) examining the possible association of differential visual acuity sighting dominance.

\section{METHOD}

The study was conducted on 180 individuals, aged 10 to 40 years, who were outpatients at the ophthalmology clinic of the Marcilio Dias Naval Hospital in Rio de Janeiro. All subjects were submitted to ophthalmological examination. The following criteria were used to include subjects in the study: (1) far and near bifoveal fixation and absence of anomalous eye movements (such as nystagmus), (2) absence of external pathologies, (3) refractometric errors of 3.00 spherical diopters or 1.50 cylindrical diopters and with differences of 0.75 spherical or cylindrical diopters between OD and OS.

Resolving power of the eye was measured with two scales (A and B) with constant $0.05^{\prime}$ variation in visual angle and covering an acuity range of 0.67 to 1.00 (scale A) and 1.00 to 2.50 (scale B). The two scales were constructed as follows: (1) at each angular level there were 10 optotypes ( $E$ of Snellen), which were randomly oriented and of constant spacing (the distance between optotypes on each line was always equal to their height); (2) the vertical spacing between rows had the same value as the height of the optotype in the lower row.
The visual threshold was taken to be the angular value of the smallest line discriminated with a maximum of four errors $(60 \%$ correct responses). All measurements were made in the same place by the same examiner, with subjects located at a distance of $6 \mathrm{~m}$ from the chart and optically corrected.

Lighting conditions were constant throughout the experiment $(800 \mathrm{~lx}$ for the chart and $750 \mathrm{~lx}$ for the surrounding environment) and were assumed to provide maximal contrast on the chart (black optotypes on white Kodagraph paper). Actually, the contrast of this type of chart (Lmax $-\mathrm{L} \min / \mathrm{L} \max +\mathrm{Lmin}$, where $\mathrm{L} \max =$ luminance of the white part of the chart, and Lmin = luminance of the black part of the optotype) is around 90\% (Lavergne \& Dehon, 1978).

Sighting dominance was determined by the test of Dolmann (Milles, 1929) as follows: a $30 \times 30 \mathrm{~cm}$ sheet of paper with a central hole $3.0 \mathrm{~cm}$ in diameter was placed on a table. The subject to be tested was then asked to hold the sheet with both hands and, keeping both arms extended, look with both eyes open toward a fixation point. Under these conditions, a cover test easily showed the eye used for fixation, which was then considered to be the dominant one.

\section{RESULTS AND DISCUSSION}

Figures 1 and 2 show the distribution of visual angles observed for OD and OS, respectively. These distributions were considered to be reasonably symmetrical by the $Z$-scores test and therefore fit to be described by means $(M)$ and standard deviations $(S D)$, with values of $M=$ 0.704 and $S D=0.100$ for $O D$ and $M=0.711$ and $S D$ $=0.104$ for OS. Analysis of the figures shows that scale A was used only once, since only one of the 360 eyes examined showed a visual acuity of 1.0 , whereas all others had visual angles of less than 1' of arc (higher than 1.0 acuity). These data clearly indicate that it is not justified to measure the differential acuity of the two eyes of normal persons with tables whose lowest angular value is equal to $1^{\prime}$ of arc, since this value does not represent maximal resolving power of the eye, but rather the lower limit of normal resolving power (Frisén, \& Frisén, 1981).

Figure 3 shows the distribution of angular differences (OS-OD) observed, which represents the quantitative expression of the phenomenon of differential acuity of the two eyes. In 80 individuals (45\% of the sample) there were no differences and in the remaining individuals there was a slight preponderance of positive differences (OS > OD visual angles), which was not statistically significant by the $t$ test for mean differences.

In any case, in $95.5 \%$ of the individuals who showed OD visual acuity differing from OS acuity the differences did not exceed $0.10^{\prime}$ of arc. On this basis, we believe that

Table 1

Relationship Between Visual Acuity and Sighting Dominance in 180 Subjects Who Were Optically Corrected and Approximately Isometropic

\begin{tabular}{cccc} 
& \multicolumn{3}{c}{ Sighting Dominance } \\
\cline { 2 - 4 } Visual & OD & OS & Total \\
\hline Acuity & 44 & 16 & 60 \\
OD > OS & 21 & 18 & 39 \\
OS > OD & 51 & 30 & 81 \\
OD $=$ OS & 116 & 64 & 180 \\
Total & 116
\end{tabular}


approximately isometropic and optically corrected eyes tend to show isoacuity. The possible differences observed are probably random; therefore, it appears that the phenomenon of visual acuity is not dependent on sighting dominance. As a matter of fact, the data (shown in Table 1) were conclusive, inasmuch as the chi-square test revealed independence between the two variables at the $10 \%$ level of significance [obtained $\chi^{2}=4.0$ and $\chi^{2}(10 \%)$ $=4.61]$.

\section{REFERENCES}

Coren, S., \& Kaplan, C. P. (1971). Patterns of ocular dominance. American Journal of Optometry \& Archives of American Academy of Optometry, 50, 283-292.

Crovitz, H. F. (1960). Differential acuity of the two eyes and the problem of ocular dominances. Science, 134, 614.

Frisén, L., \& Frisén, M. (1981). How good is normal visual acuity? Albrecht von Graefs Archiv für Klinische und Experimentelle Ophthalmology, 215, 149-157.
Gahagan, L. (1933). Visual dominance-acuity relationships. Journal of General Psychology, 9, 455-459.

Gronwall, D. M. A., Sampson, H. (1971). Ocular dominance: A test of two hypotheses. British Journal of Psychology, 62, 175-185.

Hebben, N., Benjamins, D., Milberg, W. (1981). The relationship among sighting dominance and acuity dominance in elementary school children. Cortex, 17, 441-446.

LAVERGNe, G., DehON, P. (1978). Les bases anatomophysiologigues de l'acuité visuelle. Journal Francais d'Opthalmologie, 1, 75-81.

Milles, W. (1929). Ocular dominance demonstrated by unconscious sighting. Journal of Experimental Psychology, 12, 113-126.

PorAc, C., Whitford, F. W., \& Coren, S. (1976). The relationship between eye dominance and monocular acuity: An additional consideration. American Journal of Optometry \& Physiological Optics, 53, 803-806.

Romanio, J. (1971). Vision testing. International Ophthalmology Clinics, 11, 308-316.

WALLS, G. L. (1951). A theory of ocular dominance. Archives of Ophthalmology, 45, 381-412.

(Manuscript received for publication December 10, 1987.) 